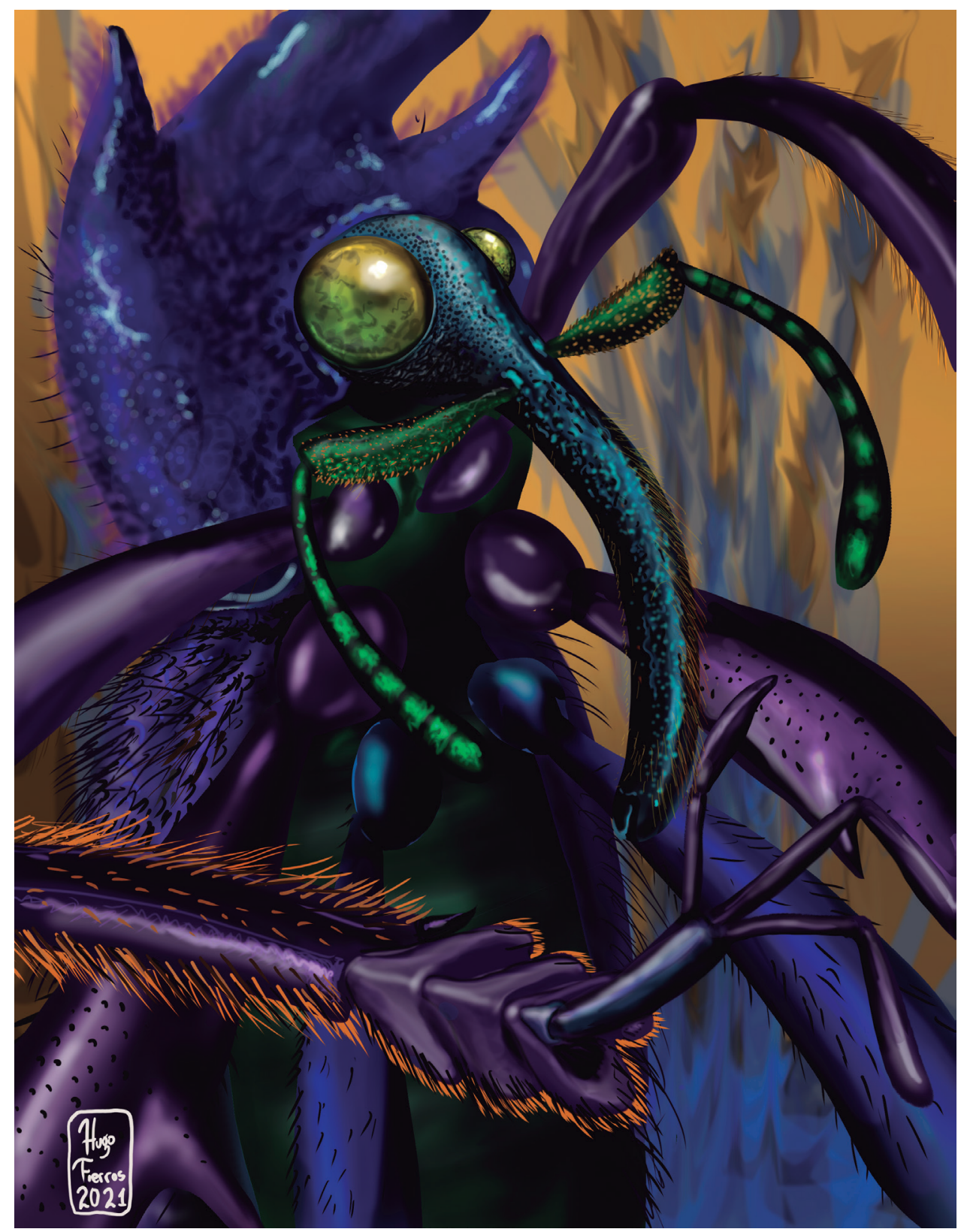

Dugesiana, Año 28, No. 2, (julio-diciembre, segundo semestre de 2021), es una publicación semestral, editada por la Universidad de Guadalajara, a través del Centro de Estudios en Zoología, por el Centro Universitario de Ciencias Biológicas y Agropecuarias. Camino Ramón Padilla Sánchez \# 2100, Nextipac, Zapopan, Jalisco, Tel. 37771150 ext. 33218, http://148.202.248.171/dugesiana/index.php/DUG/index, glenusmx@gmail.com. Editor responsable: José Luis Navarrete-Heredia. Reserva de Derechos al Uso Exclusivo 04-2009-062310115100-203, ISSN: 2007-9133, otorgados por el Instituto Nacional del Derecho de Autor. Responsable de la última actualización de este número: José Luis Navarrete-Heredia, Editor y Ana Laura González-Hernández, Asistente Editorial. Fecha de la última modificación 1 de julio de 2021, con un tiraje de un ejemplar.

Las opiniones expresadas por los autores no necesariamente reflejan la postura del editor de la publicación.

Queda estrictamente prohibida la reproducción total o parcial de los contenidos e imágenes de la publicación sin previa autorización de la Universidad de Guadalajara. 


\title{
Nuevas especies de luciérnagas (Coleoptera: Lampyridae) de México
}

\section{New fireflies (Coleoptera: Lampyridae) from México}

\author{
Santiago Zaragoza-Caballero,3*, Daniel E. Domínguez-León ${ }^{1,4}$, Mireya González-Ramírez ${ }^{1,5}$, Sara \\ López-Pérez ${ }^{2,6}$, Geovanni M. Rodríguez-Mirón ${ }^{2,7}$, Viridiana Vega-Badillo ${ }^{1,8}$, Paulina Cifuentes-Ruiz ${ }^{1,9}$ \\ ${ }^{1}$ Departamento de Zoología, Instituto de Biología, Universidad Nacional Autónoma de México, Aparta- \\ do Postal 70-153, 04510, CdMx, México; ${ }^{2}$ Colección Coleopterológica, Museo de Zoología, Facultad de \\ Estudios Superiores Zaragoza, Universidad Nacional Autónoma de México, Av. Guelatao 66, Ejército de \\ Oriente, Iztapalapa, CP 09230, CdMx, México. ${ }^{3}$ ORCID-SZC: https://orcid.org/0000-0002-0235-318X; \\ ${ }^{4}$ ORCID-DEDL: https://orcid.org/0000-0003-3848-7355; ${ }^{5}$ ORCID-MGR: https://orcid.org/0000-0002- \\ 2277-7991; ${ }^{6}$ ORCID-SLP: https://orcid.org/0000-0002-9925-0186; ${ }^{7}$ ORCID-GMRM: https://orcid. \\ org/0000-0003-0751-4672; ${ }^{8}$ ORCID-VVB: https://orcid.org/0000-0001-7064-6040; ${ }^{9}$ ORCID-PCR: ht- \\ tps://orcid.org/0000-0002-0490-7967; *Autor de correspondencia: zaragoza@ib.unam.mx
}

\section{RESUMEN}

A partir del estudio morfológico de ejemplares depositados en la Colección Nacional de Insectos, se describen e ilustran cinco nuevas especies pertenecientes a los géneros Cratomorphus Motschulsky, Tenaspis LeConte y Microphotus LeConte procedentes de Jalisco, Durango y Baja California. Las nuevas especies son Cratomorphus limai sp. nov., Tenaspis chamelensis sp. nov., Microphotus curvophallus sp. nov., Microphotus robustophallus sp. nov. y Microphotus morronei sp. nov. Además, se incluyen mapas de distribución de las especies de cada género.

Palabras clave: Diversidad, Taxonomía, Cratomorphus, Tenaspis, Microphotus.

ABSTRACT

Based on the morphological study of specimens housed at the Colección Nacional de Insectos (CNIN), five new species from Jalisco, Durango, and Baja California belonging to the genera Cratomorphus Motschulsky, Tenaspis LeConte, and Microphotus LeConte are described and illustrated. The new species are Cratomorphus limai sp. nov., Tenaspis chamelensis sp. nov., Microphotus curvophallus sp. nov., Microphotus robustophallus sp. nov., and Microphotus morronei sp. nov. In addition, a map of the distribution of the species for each genus is included.

Key words: Diversity, Taxonomy, Cratomorphus, Tenaspis, Microphotus

La familia Lampyridae está formada por 11 subfamilias, 8 tribus, 140 géneros y más de 200 especies (Braham 2010; Martin et al. 2019; Ferreira et al. 2020). En México se han descrito poco más de 200 especies incluidas en 19 géneros y 5 tribus, que representa aproximadamente el 10\% de la diversidad de lampíridos a nivel mundial. La subfamilia con mayor diversidad en el país es Lampyrinae que incluye a las tribus: Cratomorphini Green, 1948, Lamprocerini Olivier, 1907, Lamprohizini Kazantsev, 2010, Lampyrini Rafinesque, 1815 y Lucidotini Lacordaire, 1857 (Bouchard et al. 2011).

Dentro de la tribu Cratomorphini se encuentra el género Cratomorphus Motschulsky, 1853 que se caracteriza por tener cuerpo robusto, escudiforme y oblongo; pronoto ancho, semicircular, con lados explanados, ángulos posteriores redondeados y manchas translúcidas; élitros semielípticos, con el ápice redondeado y costillas marcadas; antenas cortas, filiformes, con 11 antenómeros; ojos muy grandes, ventralmente cercanos; la sutura frontoclipeal membranosa; clípeo transverso y bien diferenciado; mandíbulas ventralmente dirigidas, modificadas, expuestas, con una base casi cónica y curvadas, el ápice es reducido a un dentículo des- nudo y agudo; último palpómero maxilar fusiforme; último palpómero labial securiforme; espiráculos respiratorios torácicos tubulares; uñas con un diente en la base; abdomen con segmentos fuertemente foliados e imbricados, presentan un aparato luminoso de amplitud variable, ocupando los esternitos 5-6; borde posterior del pigidio y del último esternito variable. Hembras semejantes al macho, aladas, con el último esternito triangular y emarginado (Zaragoza et al. 2020). El género Cratomorphus se encuentra ampliamente distribuido en México (Campeche, Chiapas, Ciudad de México, Estado de México, Hidalgo, Morelos, Oaxaca, Puebla, Quintana Roo, Querétaro, San Luis Potosí, Tabasco, Veracruz y Yucatán) con 11 especies conocidas (Fig. 1).

En la tribu Lamprocerini se encuentra el género Tenaspis LeConte, 1881 que se distingue por su cuerpo marcadamente oval, escudiforme; élitros con las pleuras muy anchas; cabeza reducida, totalmente encajada en el pronoto; ojos reducidos, ampliamente separados; antenas largas, sobrepasando la mitad del cuerpo, 11 antenómeros, fuertemente comprimidos y dentados; mandíbulas modificadas con la base gruesa y pilosa, y la mitad apical en forma de estilete desnudo; clípeo libre, sutura frontoclípeal evidente; 
labro membranoso; palpos maxilares con el último artejo fusiforme, muy robusto; los labiales con el último artejo securiforme; abdomen con los esternitos y terguitos foliados e imbricados; último esternito profundamente escotado (Zaragoza-Caballero 1995). En México se conocen dos especies del género Tenaspis, las dos especies descritas se encuentran distribuidas en Chiapas, y Veracruz cuenta con el registro de una especie (Fig. 2).

El género Microphotus LeConte, 1866 se encuentra dentro de la tribu Lampyrini, se distinguen por su cuerpo elongado con los márgenes de los élitros paralelos; antenas no comprimidas, más cortas que el pronoto, compuestas de ocho a diez antenómeros con un proceso pequeño en la punta del antenómero terminal; espacio interocular cóncavo, pronoto débilmente transverso y ampliamente redondeado al frente; piezas bucales reducidas y apenas visibles entre los ojos; élitros más cortos que el abdomen; terguitos abdominales no foliados, los machos presentan un proceso lobulado o triangular medial en el penúltimo ventrito abdominal; los espiráculos se encuentran ubicados cerca de la base y ligeramente exteriores a los márgenes laterales de los ventritos II- VIII. Hasta el momento en México se conocen cuatro especies distribuidas en el norte del país (Fig. 3) (Baja California, Coahuila, Chihuahua, Durango y Sonora) (Green 1959; Cognato y Usener 2006).

La diversidad de Lampyridae a nivel mundial y en México ha ido incrementando en los últimos años. En México aún faltan muchos lugares por explorar y por tanto más especies por describir. En este trabajo se describen cinco nuevas especies correspondientes a los géneros Cratomorphus, Tenaspis y Microphotus.

\section{MATERIALES Y MÉTODOS}

Las nuevas especies se reconocieron a partir del estudio morfológico de 24 ejemplares (23 machos y 1 hembra) depositados en la Colección Nacional de Insectos (CNIN). Para la revisión de los ejemplares, su disección y la toma de medidas se utilizó un microscopio estereoscopio Carl Zeiss $^{\mathrm{TM}}$ Discovery V8. Una vez separados los genitales masculinos, se montaron en triángulos de cartón y se incorporaron al ejemplar correspondiente. La serie tipo quedo depositada en la misma colección.

Las fotografías digitales se tomaron en el Laboratorio de Microscopía y Fotografía de la Biodiversidad del Instituto de Biología, UNAM con un microscopio estereoscópico Carl Zeiss ${ }^{\mathrm{TM}}$ Axio Zoom V16, equipado con una cámara Zeiss Axiocam MRc5 y con el software ZENTM $=$ Zeiss Efficient Navigation pro-2012.

La información de la distribución se tomó de Green (1959) Zaragoza-Caballero (1995, 1996, 2012) y de ejemplares depositados en la CNIN, a partir de estos datos se elaboraron tres mapas que ilustran la distribución de las especies por género, utilizando el programa ArcGIS 10.2.2.

\section{TRATAMIENTO SISTEMÁTICO}

Cratomorphus limai Zaragoza-Caballero, Domínguez-León y González-Ramírez sp. nov.

Figura 4 A-E

http://zoobank.org/A02BAA93-C8DA-4CEF-857D913171B43D0A

Macho. Largo $15.08 \mathrm{~mm}$; ancho $6.84 \mathrm{~mm}$. Cuerpo ancho, escudiforme, oblongo, de color negro a excepción del pronoto, escutelo, margen lateral y sutural de los élitros, pro y mesotórax; trocánteres y parte basal de los fémures amarillos; pronoto con dos manchas translúcidas (Fig. 4A). Aparato luminoso ocupando la parte media de los esternitos 5-6 (Fig. 4B).

Cabeza (Fig. 4B). Espacio interocular $(0.53 \mathrm{~mm})$ profundamente excavado, opaco y espaciadamente piloso; frente vertical; distancia interantenal reducida a la cuarta parte de lo ancho de la fosita antenal $(0.52 \mathrm{~mm})$; ojos finamente facetados, muy desarrollados, semiesféricos, más largos que anchos (1.48 mm X $1.22 \mathrm{~mm}$ ); antenas filiformes, cortas, un poco más largas que el pronoto $(4.92 \mathrm{~mm}$ X $3.63 \mathrm{~mm}$ ), sobrepasando apenas las mesocoxas, escapo claviforme, un poco más largo que los dos antenómeros siguientes juntos $(0.77 \mathrm{~mm})$, el segundo corto $(0.31 \mathrm{~mm})$, el tercero de $0.53 \mathrm{~mm}$, del cuarto al octavo de $0.43 \mathrm{~mm}$, el noveno y décimo de $0.37 \mathrm{~mm}$ y el undécimo alcanza 0.47 mm de longitud; sutura frontoclipeal membranosa, cóncava; clípeo trapezoidal, con el borde anterior recto, superficie con sedas largas regularmente distribuidas; mandíbulas robustas, con abundantes sedas externas y terminadas en un estilete agudo; labro membranoso; palpómero maxilar apical romboidal; el labial en forma securiforme.

Tórax (Fig. 4A-B). Pronoto más ancho que largo (5.42 $\mathrm{mm} \times 3.63 \mathrm{~mm}$ ), semicircular, borde posterior recto, ángulos posteriores redondeados, disco convexo, lados anchamente explanados, superficie opaca, porosa, con una diminuta y abundante pilosidad decumbente; escutelo triangular, superficie plana y pilosa, con el borde posterior agudo; élitros oblongos, más de tres veces más largos que anchos (11.45 $\mathrm{mm} \times 3.42 \mathrm{~mm})$, superficie rugosamente punteada, opaca y con pilosidad procumbente; espiráculos respiratorios meso-torácicos membranosos; patas semejantes entre sí, fémures fusiformes, aplanados, tibias aplanadas, un poco dilatadas hacia el ápice, borde externo entero, tarsómeros lateralmente no comprimidos, primer metatarsómero ligeramente más largo que los dos siguientes juntos $(0.53 \mathrm{~mm}$ $\times 0.47 \mathrm{~mm}$ ), el cuarto bífido, cubriendo más de la mitad del quinto, uñas simples.

Abdomen (Fig. 4B). Con siete esternitos visibles, el último fuertemente mucronado. Tergitos foliados e imbricados, borde posterior del pigidio truncado; espiráculos abdominales dorsalmente abiertos; edeago robusto (Fig. 4C-D), casi cilíndrico, con la pieza basal reducida, lóbulos laterales no soldados, triangulares, cubriendo casi por completo al lóbulo medio en su parte dorsal, con el ápice recurvado, parte dorsal esclerosada y la ventral membranosa, orificio medio terminal.

Variación. Únicamente en la talla que va desde los 15.08 $\mathrm{mm}$ a $16.36 \mathrm{~mm}$ de largo y el ancho va de $6.84 \mathrm{~mm}$ a 7.09 $\mathrm{mm}$. 
Etimología. Especie dedicada a William Lima, distinguido estudiante dedicado a la revisión de la tribu Cratomorphini (Coleoptera: Lampyridae).

Material examinado. Holotipo macho etiquetado: México, Jalisco, Casimiro Castillo, El Pacotal, $600 \mathrm{msnm}$, BTSC. 23/III/1996, col. J. Ascencio. Paratipo macho con mismos datos.

\section{Tenaspis chamelensis Zaragoza-Caballero, López-Pérez y Rodríguez-Mirón sp. nov. Figura 5 A-E \\ http://zoobank.org/5CE843BC-369A-40BA-8F55- E6888AE0302E}

Macho. Largo $11.09 \mathrm{~mm}$; ancho $5.27 \mathrm{~mm}$. Cuerpo ancho, escudiforme, oblongo, pronoto amarillento con tres manchas oscuras; élitros café obscuro, con dos bandas humerales amarillas divergentes (Fig. 5A); cabeza, antenas, pro, meso y metatórax, patas además del pigidio y último esternito moreno obscuro; abdomen amarillo; sin aparato luminoso (Fig. 5B).

Cabeza (Fig. 5B). Espacio interocular $(0.36 \mathrm{~mm})$ casi plano, opaco y espaciadamente piloso; frente vertical; distancia interantenal reducida a la mitad de lo ancho de la fosita antenal $(0.12 \mathrm{~mm})$; ojos finamente facetados, reducidos, semiesféricos, casi tan largos como anchos (0.36 $\mathrm{mm}$ X $0.31 \mathrm{~mm}$ ); antenas serradas, largas, 2.25 veces más largas que el pronoto, sobrepasando las coxas posteriores, escapo claviforme, un poco más largo que los dos antenitos siguientes juntos $(0.46 \mathrm{~mm})$, el segundo corto $(0.12 \mathrm{~mm})$, del tercero de $0.25 \mathrm{~mm}$, del cuarto al octavo de $0.46 \mathrm{~mm}$, el noveno de $0.55 \mathrm{~mm}$, el décimo de $0.65 \mathrm{~mm}$ y el undécimo alcanza $0.93 \mathrm{~mm}$ de longitud; sutura frontoclipeal marcada por una línea recta; clípeo trapezoidal, con el borde anterior cóncavo, superficie con sedas largas regularmente distribuidas; mandíbulas curvadas, robustas, con abundantes sedas externas y terminadas en un estilete agudo; labro membranoso; palpómero maxilar apical romboidal; el labial en forma de bumerán.

Tórax (Fig. 5A-B). Pronoto más ancho que largo (4.24 $\mathrm{mm} \times 2.4 \mathrm{~mm}$ ), un tanto triangular, ápice redondeado, borde posterior ondulado, ángulos posteriores redondeados, disco convexo, con una quilla anterior, lados anchamente explanados, superficie brillante, rugosamente porosa y con una diminuta pilosidad decumbente; escutelo triangular con el borde posterior agudo y piloso, superficie brillante, convexa, diminutamente punteada; élitros oblongos, casi tres veces más largos que anchos $(9.27 \mathrm{~mm} \times 3.36 \mathrm{~mm})$, superficie rugosamente punteada, opaca, con dos tipos de pilosidad, una relativamente larga y erecta, la otra muy pequeña y procumbente; epipleura ancha; espiráculos respiratorios meso-torácicos membranosos; patas semejantes entre sí, fémures fusiformes, aplanados, tibias aplanadas, un poco dilatadas al ápice, borde externo entero, tarsómeros lateralmente comprimidos, primer metatarsómero ligeramente más largo que los dos siguientes juntos $(0.53 \mathrm{~mm} \times 0.51$ $\mathrm{mm}$ ), el cuarto bífido, cubriendo más de la mitad del quinto, uñas simples.

Abdomen (Fig. 5B). Con siete esternitos visibles, el último fuertemente escotado. Tergitos foliados e imbricados, borde posterior del pigidio excavado; espiráculos abdominales ventro- lateralmente abiertos; edeago robusto (Fig. 5C-E), casi circular, con la pieza basal reducida a $2 / 3$ de los lóbulos laterales, éstos con la porción terminal filamentosa y soldados al lóbulo medio, en la base dorsal; lóbulo medio apicalmente dilatado y terminado en triángulo, con la parte dorsal esclerosada y la ventral membranosa, orificio medio terminal.

Hembra. Semejante al macho. Alada, con antenas aplanadas, menos serradas, pigidio y último esternito triangular

Variación. Únicamente en la talla que va de $11.09 \mathrm{~mm}$ a $14.0 \mathrm{~mm}$ de largo y del ancho va de $5.27 \mathrm{~mm}$ a $7.27 \mathrm{~mm}$.

Etimología. El epíteto alude al lugar de procedencia.

Material examinado. Holotipo macho etiquetado: México, Jalisco. Estación de Biología Chamela 29/07/2002, col S. Zaragoza. Serie de paratipos: Misma localidad 1 hembra 20-27/ VIII/1984, cols. J.A. Chemsak y T.J. Doyen at lights; 1 macho 1/VIII/1985, col F. Noguera; 1 macho 24/ VIII/1985, col. M. Sánchez; 2 machos 4-9/IX/1992, col. A. Rodríguez; 1 macho 1/VIII/1994, col. R. Ayala; 1 macho 15-20/VII/1999, col. A. Rodríguez; 1 macho 15/VIII/1998, col. E. Ramírez; 1 macho 19-24/VII/2000, col. A. Rodríguez.

Microphotus curvophallus Zaragoza-Caballero, Vega-Badillo y Cifuentes-Ruiz sp. nov.

Figuras 6 A-B, 7 A-C

http://zoobank.org/D0BCED73-C181-4A5A-A2C75441EA66E5FC

Macho. Largo $4.80 \mathrm{~mm}$; ancho $1.68 \mathrm{~mm}$. Cuerpo ancho, oblongo, pronoto cremoso, con una difusa mancha café (Fig. 6A); élitros, tórax, patas y abdomen negro pálido antenas, frente y palpos de color ambarino. Sin aparato luminoso (Fig. 6B).

Cabeza (Fig. 6B). Espacio interocular $(0.24 \mathrm{~mm})$ profundamente excavado; frente vertical; distancia interantenal reducida a la mitad de lo ancho de la fosita antenal (0.05 $\mathrm{mm})$; ojos facetados, muy grandes, esféricos, más largos que anchos ( $0.79 \mathrm{~mm} \mathrm{X} 0.71 \mathrm{~mm})$; antenas muy cortas, casi moniliformes, de ocho antenómeros, escapo claviforme de $0.18 \mathrm{~mm}$, el primero más corto que los dos siguientes juntos $(0.23 \mathrm{~mm})$, el segundo globular de $0.8 \mathrm{~mm}$, el tercero triangular de $0.15 \mathrm{~mm}$, los cinco siguientes casi de la misma longitud, alcanzando en conjunto $0.47 \mathrm{~mm}$, el octavo con un pequeño tubérculo membranoso; sutura frontoclipeal marcada por una línea; clípeo romboidal, con el borde anterior agudo, con sedas esclerosadas al ápice; mandíbulas cortas, robustas, ligeramente curvadas, con abundantes sedas externas; labro membranoso; palpómero maxilar apical reducido, romboidal; el labial fusiforme.

Tórax (Fig. 6A-B). Pronoto tan ancho como largo (1.45 $\mathrm{mm}$ ), con los lados posteriormente convergentes, ápice redondeado, borde posterior cóncavo, ángulos posteriores romos, disco convexo, espaciadamente punteado y piloso; escutelo espatulado, con el borde posterior truncado; superficie convexa, espaciadamente pilosa; élitros tres veces más largos que anchos (3.16 X $1.04 \mathrm{~mm})$ rugosamente punteados y pilosos, pilosidad procumbente, epipleura angosta; espiráculos respiratorios torácicos membranosos; patas 
semejantes entre sí, fémures fusiformes, aplanados, tibias aplanadas y dilatadas al ápice, metatrocánter largo casi un tercio del metafémur; primer metatarsómero corto, un poco más de la mitad de los tres siguientes juntos $(0.18 \mathrm{~mm} \mathrm{X}$ $0.31 \mathrm{~mm}$ ), el cuarto lobulado, dejando casi totalmente descubierto al quinto, uñas simples.

Abdomen (Fig. 6B). Con siete esternitos visibles, el último fuertemente mucronado; tergitos foliados e imbricados, espiráculos abdominales ventro- lateralmente abiertos; edeago robusto (Fig. 7A-C), pieza basal casi tan larga como los lóbulos laterales, estos triangulares, con el ápice recurvado y expansiones internas, posteriormente proyectadas; lóbulo medio lateralmente comprimido, muy curvado, con el ápice romo y el orificio medio terminal.

Comentarios taxonómicos: La talla reducida -menos de $6.00 \mathrm{~mm}$ - y en general la apariencia, lo relaciona con $\mathrm{Mi}$ crophotus octarthrus Fall, 1912 de la que se separa por la forma del edeago, sobre todo la curvatura semicircular que presenta el lóbulo medio. En M. octarthrus es sigmoide.

Etimología. El epíteto curvophallus está compuesta por los vocablos del latín curvus = curvo y phallus = pene, la cual refiere a la curvatura semicircular que presenta el lóbulo medio.

Material examinado. Holotipo macho etiquetado: México, Durango, Peñón Blanco (Belém), 10/VII/1985, cols. H. Brailovsky y E. Barrera.

Microphotus robustophallus Zaragoza-Caballero, Domínguez-León y González-Ramírez sp. nov. Figuras 6 C-D, 7 D-F

http://zoobank.org/97FC3A3F-6794-4DAF-B13014D2830EA721

Macho. Largo $7.49 \mathrm{~mm}$; ancho $2.26 \mathrm{~mm}$. Cuerpo café claro, oblongo, pronoto cremoso, con una mancha morena basal; escutelo cremoso (Fig. 6C); sin aparato luminoso (Fig. 6D).

Cabeza (Fig. 6C). Espacio interocular $(0.27 \mathrm{~mm})$ profundamente excavado; frente vertical; distancia interantenal reducida a más de la mitad de lo ancho de la fosita antenal $(0.04 \mathrm{~mm})$; ojos facetados, muy grandes, esféricos, más largos que anchos ( $0.85 \mathrm{~mm}$ X $0.77 \mathrm{~mm})$; antenas muy cortas, casi moniliformes, de nueve antenómeros; escapo claviforme de $0.22 \mathrm{~mm}$, más corto que los dos siguientes juntos $(0.36 \mathrm{~mm})$, el segundo casi cuadrado de $0.13 \mathrm{~mm}$, el tercero triangular de $0.23 \mathrm{~mm}$, los cinco siguientes casi de la misma longitud, alcanzando en conjunto $0.53 \mathrm{~mm}$, el noveno con un pequeño tubérculo membranoso; sutura frontoclipeal marcada por una línea; clípeo romboidal, con el borde anterior truncado; con sedas irregularmente distribuidas; mandíbulas cortas, robustas, sin sedas externas; labro membranoso; palpómero maxilar apical reducido, romboidal; el labial securiforme.

Tórax (Fig. 6D-E). Pronoto más ancho que largo (2.50 $\mathrm{mm}$ X $2.04 \mathrm{~mm}$ ), con los lados redondeados, más amplios en la parte media, ápice redondeado, borde posterior cóncavo, ángulos posteriores casi rectos, disco deprimido, con un surco medio, rugosamente punteado y piloso; escutelo espatulado, finamente punteado y piloso, con el borde posterior redondeado; élitros más de cuatro veces y media más largos que anchos (5.45 $\mathrm{mm} \mathrm{X} 1.13 \mathrm{~mm}$ ) rugosamente punteados y pilosos, pilosidad procumbente, epipleura angosta; espiráculos respiratorios torácicos tubulares; patas semejantes entre sí, fémures fusiformes, aplanados, tibias aplanadas, dilatadas al ápice, metatrocánter largo casi un tercio del metafémur; primer metatarsómero tan largo como los tres siguientes juntos (0.26), el cuarto lobulado, dejando casi totalmente descubierto al quinto, uñas simples.

Abdomen (Fig. 6D). Con siete esternitos visibles, el último fuertemente mucronado; tergitos no foliados, espiráculos abdominales ventro-lateralmente abiertos; edeago robusto (Fig. 7D-F), pieza basal corta, alcanzando la mitad de los lóbulos laterales, éstos triangulares, con el ápice recurvado, dorsalmente cubriendo al lóbulo medio, sedosas en su borde interno, ventralmente, las expansiones internas se alojan en concavidades del lóbulo medio; el lóbulo medio muy robusto en su base, con la parte terminal comprimida y roma, orificio medio terminal.

Comentarios taxonómicos. La presencia de nueve antenómeros relaciona esta especie con Microphotus morronei sp. nov., de la que se separa por tener: el pronoto más plano, con un surco medio, y el lóbulo medio del edeago es mucho más robusto, y no longitudinalmente comprimido.

Etimología. La palabra robustophallus está compuesta por los vocablos del latín robustus = robusto y phallus = pene, la cual refiere a la robustez del lóbulo medio del edeago.

Material examinado. Holotipo macho etiquetado: México, Baja California Sur, El Triunfo, $15.2 \mathrm{~km} \mathrm{S,} 24.5 \mathrm{E}$ (23.67406 N; $110.09121 \mathrm{~W}), 543 \mathrm{msnm}, 20 / \mathrm{IX} / 2012$, col. E. Ríos.

Microphotus morronei Zaragoza-Caballero, López-Pérez y Rodríguez-Mirón sp. nov. Figura 6 E-F, 7 G-I

http://zoobank.org/56BFA735-AB3A-42D6-AEA14FC79022A292

Macho. Largo $7.40 \mathrm{~mm}$; ancho $2.72 \mathrm{~mm}$. Cuerpo moreno claro, oblongo, pronoto cremoso, con una mancha morena basal, emarginada; escutelo cremoso; márgenes elitrales más amarillos (Fig. 6E); sin aparato luminoso (Fig. 6F).

Cabeza (Fig. 6F). Espacio interocular $(0.34 \mathrm{~mm})$ profundamente excavado; frente vertical; distancia interantenal más de la mitad de lo ancho de la fosita antenal (0.08 X $0.13 \mathrm{~mm}$ ); ojos facetados, muy grandes, esféricos, más largos que anchos (1.27 mm X $0.72 \mathrm{~mm})$; antenas muy cortas, casi moniliformes, de nueve antenómeros; escapo claviforme de $0.21 \mathrm{~mm}$, casi de la mitad que los dos siguientes juntos $(0.41 \mathrm{~mm})$, el segundo casi cuadrado de $0.15 \mathrm{~mm}$, el tercero triangular de $0.26 \mathrm{~mm}$, los cinco siguientes casi de la misma longitud, alcanzando en conjunto $0.50 \mathrm{~mm}$, el noveno con un pequeño tubérculo membranoso; sutura frontoclipeal marcada por una línea; clípeo romboidal, con el borde anterior romo; con sedas irregularmente distribuidas; mandíbulas cortas, robustas, curvadas, sin sedas externas; labro membranoso; palpómero maxilar apical reducido, romboidal; el labial fusiforme.

Tórax (Fig. 6E-F). Pronoto más ancho que largo (1.90 
$\mathrm{mm}$ X $2.72 \mathrm{~mm}$ ), con los lados redondeados, más amplios en la parte media, ápice redondeado, borde posterior cóncavo, ángulos posteriores casi rectos, disco deprimido, rugosamente punteado y piloso; escutelo espatulado, finamente punteado y piloso, con el borde posterior redondeado; élitros poco más de tres veces y media más largos que anchos (4.68 $\mathrm{mm} \mathrm{X} 1.36 \mathrm{~mm})$ rugosamente punteados, pilosos, con dos costillas longitudinales, pilosidad procumbente, epipleura angosta; espiráculos respiratorios torácicos membranosos; patas, semejantes entre sí, fémures fusiformes, aplanados, tibias aplanadas, dilatadas al ápice, metatrocánter corto casi un cuarto de lo largo del metafémur; primer metatarsómero más de la mitad de largo que los tres siguientes juntos $(0.25 \mathrm{~mm}$ X $0.37 \mathrm{~mm})$, el cuarto lobulado, dejando casi totalmente descubierto al quinto, uñas simples.

Abdomen (Fig. 6F). Con siete esternitos visibles, el último con el mucrón corto y redondeado; tergito foliados, espiráculos abdominales ventro-lateralmente abiertos; edeago robusto (Fig. 7G-I), pieza basal corta, alcanzando la mitad de los lóbulos laterales, éstos triangulares, con el ápice redondeado, dorsalmente dejando al descubierto al lóbulo medio, con expansiones posteriormente proyectadas en su borde interno; el lóbulo medio comprimido, con la parte terminal aguda, orificio medio terminal.

Comentarios taxonómicos. Microphotus morronei sp. nov. se parece a $M$. dilatatus; sin embargo, difiere en tener antenas con nueve antenómeros; élitros con dos costillas; ápice de los lóbulos laterales largos y agudo, proyecciones internas redondeadas, posteriormente dirigidas, lóbulo medio ligeramente curvado, con el ápice agudo. En M. dilatatus hay ocho antenómeros; élitros sin costillas; los lóbulos laterales más cortos y terminación más ancha; las proyecciones son triangulares y anteriormente dirigidas; el lóbulo medio se curva en forma de $\mathrm{S}$ y el ápice es romo.

Etimología. Especie dedicada al Dr. Juan José Morrone Lupi, distinguido entomólogo mexicano especialista en la familia Curculionidae.

Material examinado. Holotipo macho etiquetado: México, Baja California Sur, Las Barracas, ca. $30 \mathrm{~km}$ E Santiago 1-7/X/1982. Col. Paul DeBach, Malaise tramp. depositado en la CNIN. Serie de Paratipos: Nueve machos con los mismos datos.

\section{AGRADECIMIENTOS}

Agradecemos a José Luis Navarrete-Heredia por su invitación a esta contribución que rinde reconocimiento a la labor desarrollada por el Dr. Juan José Morrone Lupi en el estudio de la familia Curculionidae, pero sobre todo por sus aportaciones en el área de la biogeografía. A la Biól. Susana Guzmán por la asistencia técnica en la toma de las fotografías. Agradecemos de igual manera a los revisores anónimos que con sus comentarios mejoraron este escrito.

\section{LITERATURA CITADA}

Bouchard, P., Y. Bousquet, A.E. Davies, M.A. AlonsoZarazaga, J. F. Lawrence,C.H.C. Lyal, A.F. Newton, C.A.M. Reid, M. Schmitt, S.A. Slipinski, and A.B.T. Smith. 2011. Family- group names in Coleoptera (Insecta). Zookeys, (88): 1-972.

Branham, M.A. 2010. Lampyridae Latreille, 1817. (pp. 141-149). In: Leschen, R.A.B., R.G. Beutel and J.F. Lawrence (Eds.). Coleoptera, Beetles. Volume 2: Morphology and Systematics (Elateroidea, Bostrichiformia, Cucujiformia partim). Walter de Gruyter, Berlin, Germany.

Cognato, A. and J. Usener. 2006. Phylogenetic review of desert firefly taxonomic characters (Coleoptera: Lampyridae: Microphotus). Insect Systematics \& Evolution, 37(1): 71-80.

Ferreira, V.S., O. Keller and M. A. Branham 2020. Multilocus phylogeny support the nonbioluminescent firefly Chespirito as a new Subfamily in the Lampyridae (Coleoptera: Elateroidea). Insect Systematics and Diversity, 4(6): 1-13.

Green, J.W. 1959. Revision of the species of Microphotus, with an emendation of the Lampyrini (Lampyridae). The Coleopterist Bulletin, 13(3): 80-96.

Martin G.J., K.F. Stanger-Hall, M.A. Branham, L.F.L. Da Silveira, S.E. Lower, D.W. Hall, X. Li, A.R. Lemmon, E.M. Lemmon and S.M. Bybee. 2019. Higher-Level phylogeny and reclassification of Lampyridae (Coleoptera: Elateroidea). Insect Systematics and Diversity, 3(6): 11-15.

Zaragoza-Caballero, S. 1995. La familia Lampyridae (Coleoptera) en la Estación de Biología Tropical "Los Tuxtlas", Veracruz, México. Instituto de Biología, Universidad Nacional Autónoma de México. Publicaciones Especiales 14.

Zaragoza-Caballero, S. 1996. Cantharoidea de México. I. Nuevas especies de Photinus (Coleoptera: Lampyridae: Photinini). Anales del Instituto de Biología. Serie Zoología, 67(2): 319-329.

Zaragoza-Caballero, S. 2012. Cratomorphus halffteri, a new species of Mexico (Coleoptera: Lampyridae: Cratomorphini). Dugesiana, 18(2): 175-179.

Zaragoza-Caballero, S., S. López-Pérez, V. Vega-Badillo, D.E. Domínguez-León, G.M. Rodríguez-Mirón, M. González-Ramírez, I.G. Gutiérrez-Carranza, P. Cifuentes-Ruiz y M.L. Zurita-García. 2020. Luciérnagas del centro de México (Coleoptera: Lampyridae): descripción de 37 especies nuevas. Revista Mexicana de Biodiversidad, 91(e913104): 1-70.

Recibido: 25 abril 2021

Aceptado: 12 junio 2021 
1

- Cratomorphus anitae

- Cratomorphus ayalai

- Cratomorphus concolor

- Cratomorphus elevatus

- Cratomorphus halfferi

- Cratomorphus hoffmannae

- Cratomorphus huautlaensis

- Cratomorphus limai sp. nov.

- Cratomorphus picipennis

- Cratomorphus ramirezi

- Cratomorphus rodriguezae

- Cratomorphus signativentris

Altitude

$0-1000 \mathrm{msnm}$

$1000-2000 \mathrm{msnm}$

$2000-3000 \mathrm{msnm}$

3000 - $4000 \mathrm{msnm}$

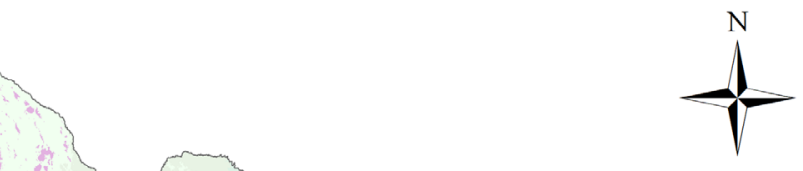

Figura 1. Mapa de distribución de las especies de Cratomorphus Motschulsky en México.

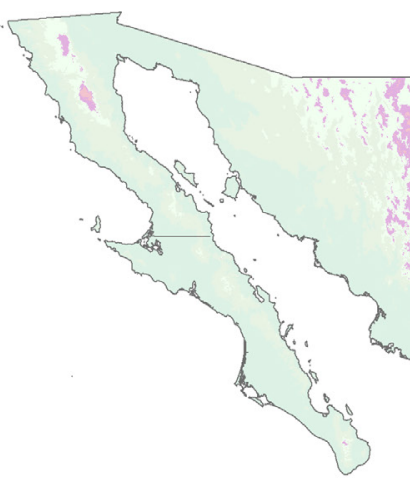

- Tenaspis angularis

- Tenaspis chamelensis sp. nov.

- Tenaspis gonzalenzis

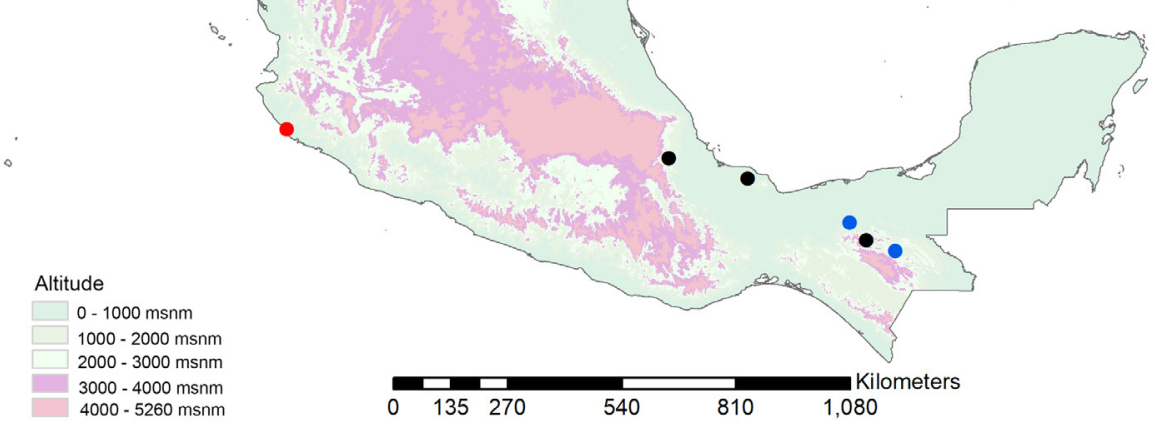

Figura 2. Mapa de distribución de las especies de Tenaspis LeConte en México. 


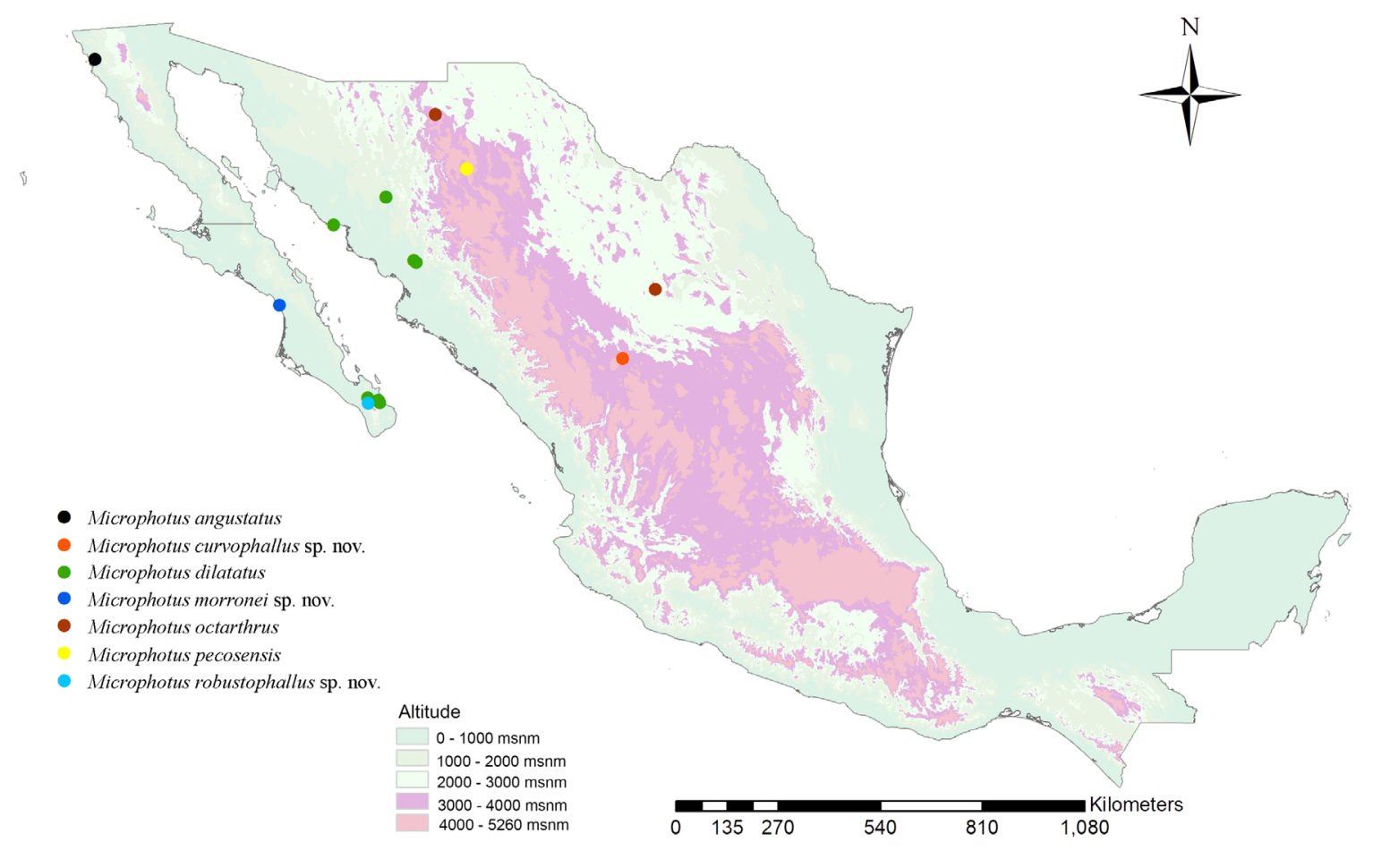

Figura 3. Mapa de distribución de las especies de Microphotus LeConte en México. 


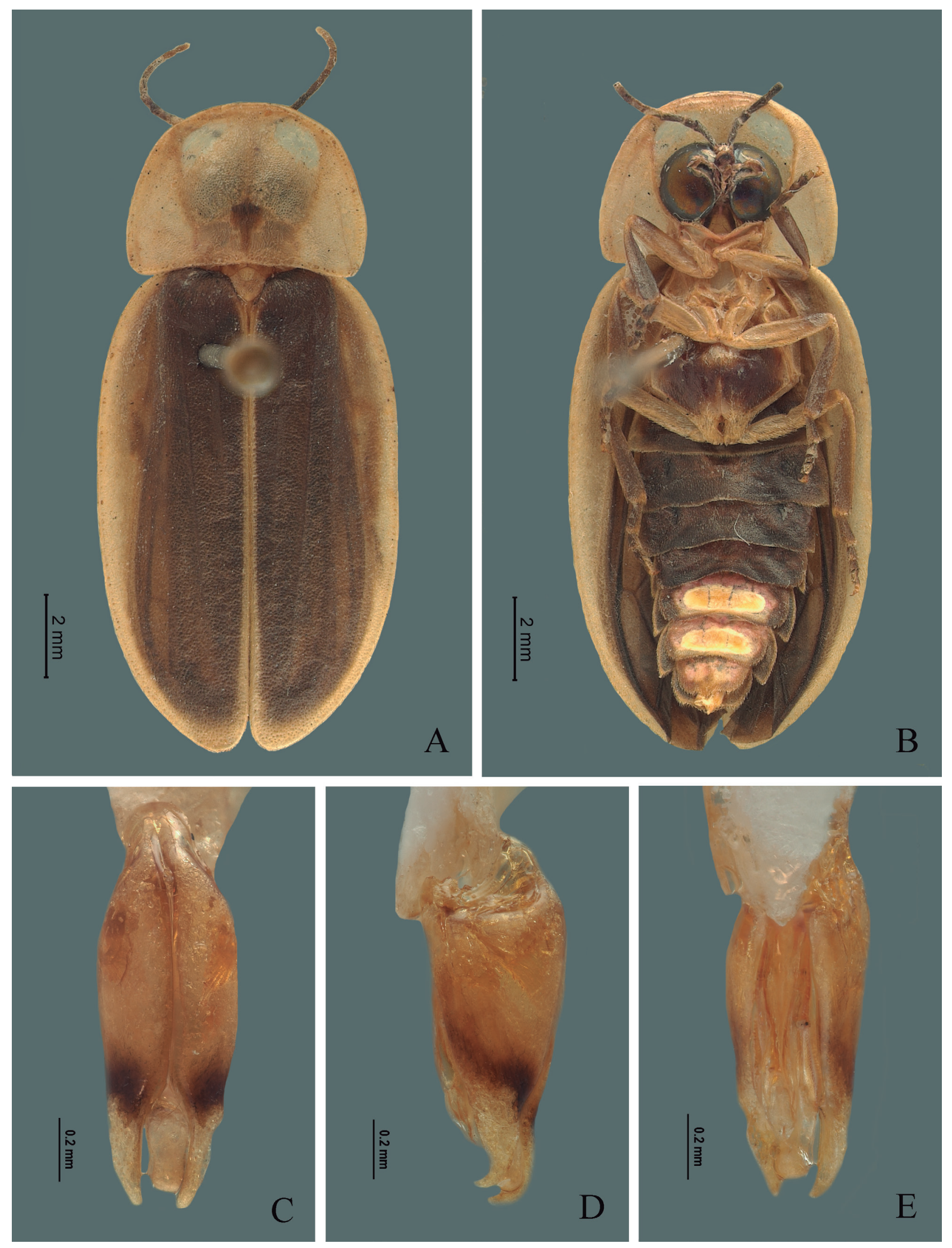

Figura 4. Cratomorphus limai sp. nov. A) Vista dorsal, B) vista ventral, C) edeago vista dorsal, D) edeago vista lateral, E) edeago vista ventral. 

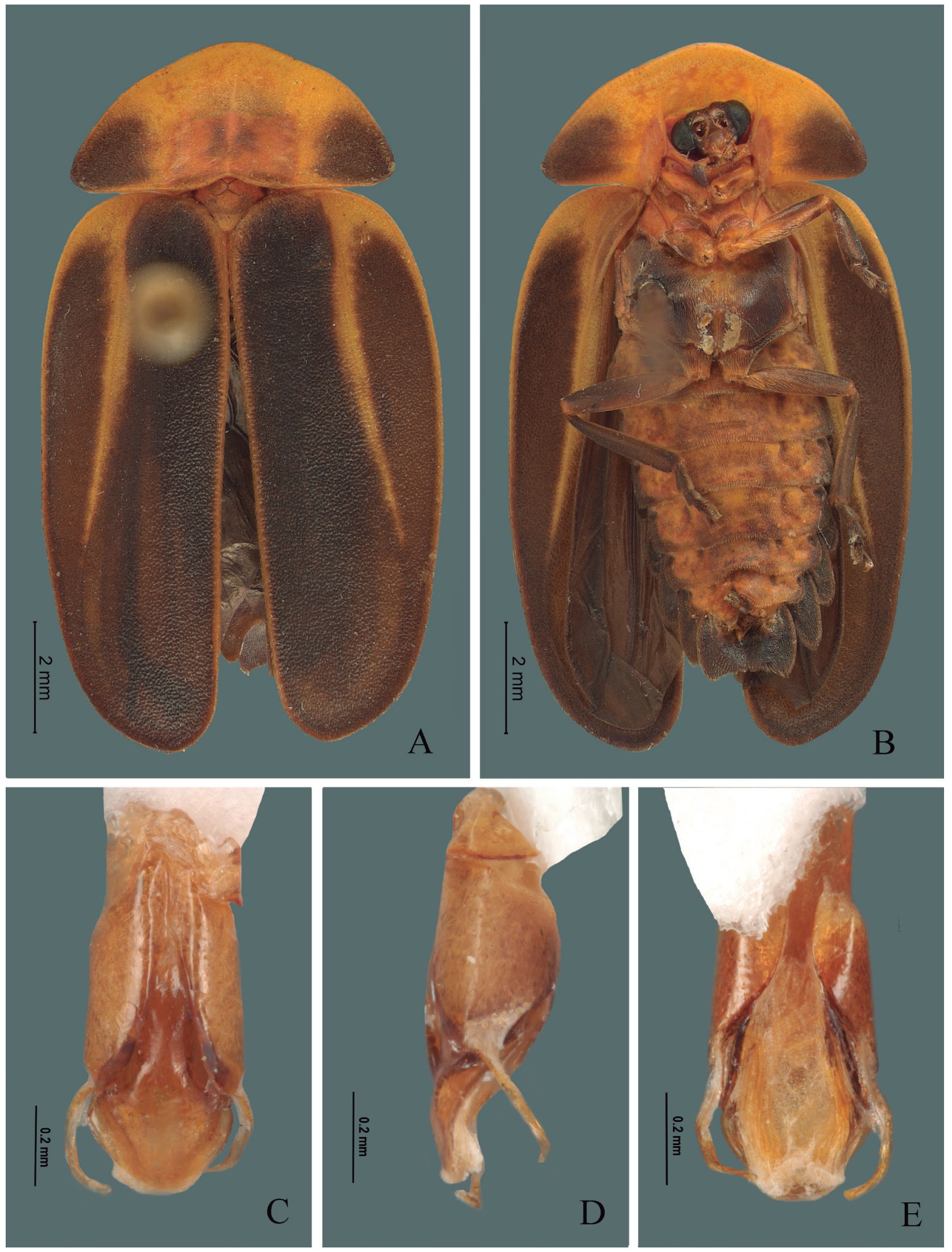

Figura 5. Tenaspis chamelensis sp, nov. A) Vista dorsal, B) vista ventral, C) edeago vista dorsal, D) edeago vista lateral, E) edeago vista ventral. 

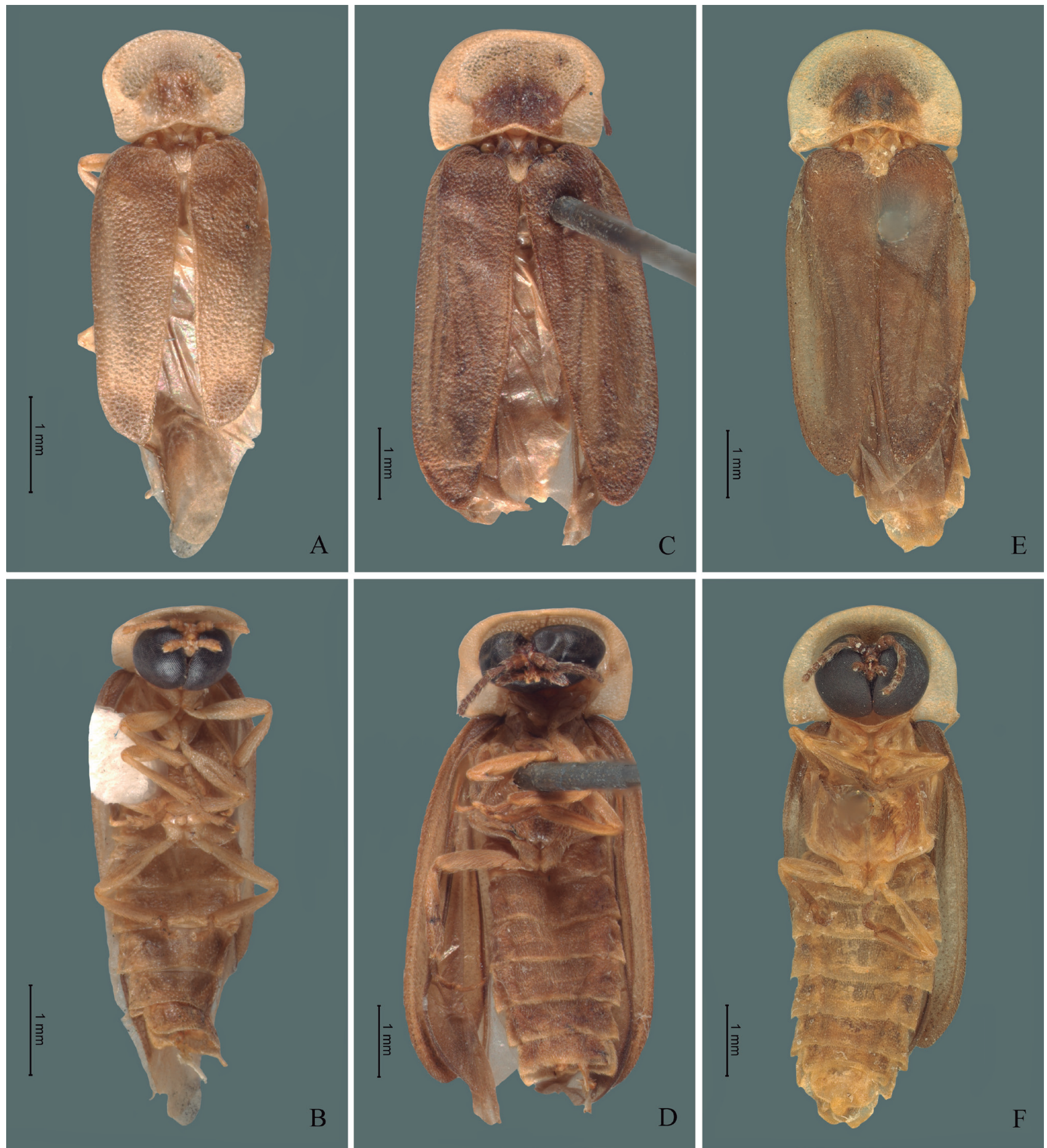

Figura 6. Nuevas especies del género Microphotus LeConte. Microphotus curvophallus sp. nov. A) Vista dorsal, B) vista ventral; Microphotus robustophallus sp. nov. C) Vista dorsal, D) vista ventral; Microphotus morronei sp. nov. E) Vista dorsal, F) vista ventral. 

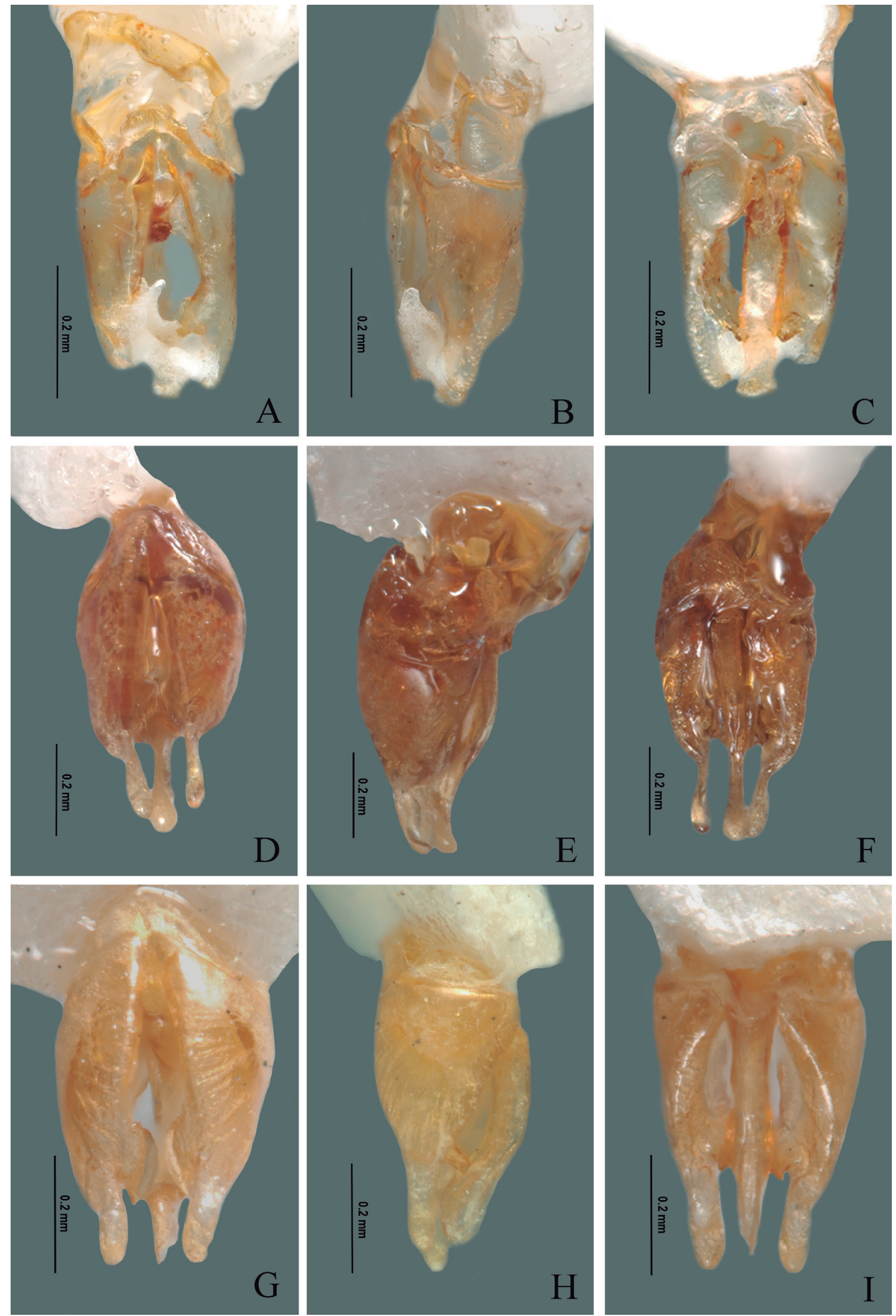

Figura 7. Edeago de las especies del género Microphotus LeConte. Microphotus curvophallus sp. nov. A) vista dorsal, B) vista lateral, C) vista ventral; Microphotus robustophallus sp. nov. D) vista dorsal, E) vista lateral, F) vista ventral; Microphotus morronei sp. nov. G) vista dorsal, H) vista lateral, I) vista ventral. 